\title{
Microshear bond strength of conventional and self-adhesive resin cements to feldsphatic ceramic
}

Estéfani Maminis Soares Couto', Bruna de Oliveira Reis², Thaís Yumi Umeda Suzuki³, Wirley Gonçalves Assunção4, André Luiz Fraga Briso ${ }^{5}$, Paulo Henrique dos Santos ${ }^{4}$.

${ }^{1}$ DDS. Department of Dental Materials and Prosthodontics, Araçatuba School of Dentistry, São Paulo State University - UNESP, Araçatuba, São Paulo, Brazil;

${ }^{2}$ DDS, MS, PhD Student. Department of Restorative Dentistry, Araçatuba School of Dentistry, São Paulo State University - UNESP, Araçatuba São Paulo, Brazil;

${ }^{3} \mathrm{DDS}, \mathrm{MS}, \mathrm{PhD}$, Post-doctoral Student. Department of Dental Materials and Prosthodontics, Araçatuba School of Dentistry, São Paulo State University - UNESP, Araçatuba, São Paulo, Brazil;

4. Associate Professor, Department of Dental Materials and Prosthodontics, Araçatuba School of Dentistry, São Paulo State University - UNESP, Araçatuba, São Paulo, Brazil.

5. Associate Professor, Department of Restorative Dentistry, Araçatuba School of Dentistry, São Paulo State University - UNESP, Araçatuba, São Paulo, Brazil.

Corresponding Author: Paulo Henrique Dos Santos Address: José Bonifácio St, 1193. Araçatuba-SP, Brazil. Zip Code: 16015-050

Phone number: 5518 3636-2802

Facsimile number: 5518 3636-3245

E-mail: paulosantos@foa.unesp.br

Received: November 17, 2017

Accepted: February 15, 2018
Aim: The aim of this study was to verify the microshear bond strength of conventional and self-adhesive resin cements to feldsphatic ceramic. Methods: Twenty discs of Starlight ceramic (Degudent) were made $(15 \mathrm{~mm} \times 2 \mathrm{~mm})$. The bonding procedure was accomplished by insertion of resin cements into tubes of $0.7 \mathrm{~mm}$ internal diameter in contact with the ceramic. The resin cements used were: RelyX ARC (3M Espe), Panavia F (Kuraray), RelyX Unicem (3M Espe) and seT (SDI). Total of six tubes of each material on each ceramic disc. The specimens were tested for microshear in a universal testing machine, 24hours and 6months after bonding procedure. Values of microshear bonding strength $(\mathrm{MPa})$ were subjected to ANOVA and Fisher PLSD test $(p<0.05)$. Results: For 24hours analysis, RelyX ARC showed the highest microshear bonding strength without statistical difference to RelyX Unicem and seT. Panavia F showed the lowest values of microshear bonding strength in both periods. In the analysis after 6 months there was a significant increase in the microshear bonding strength values for all cements compared to 24hours. Only for RelyX ARC, the increase was not statistically significant. Conclusion: In conclusion, the bond strength depends mainly on the type of resin cement used, and the self-adhesive cements behave similarly to conventional resin cement.

Keywords: Resin cements. Ceramics. Dentin-Bonding Agents. Shear Strength. 


\section{Introduction}

The restorative dentistry has been changed in the last years, with significant improvement in the esthetic issue, biocompatibility and strength of direct and indirect restorative materials ${ }^{1}$. Dental ceramics has been the choice material for aesthetic restorations because of their optical properties. The chemical stability and resistance to wear make the ceramic the indicated for both anterior and posterior areas, in which aesthetic demand and strength are necessary². Traditionally, dental porcelain is composed by two distinct phases: crystalline phase, responsible for strength, and a glass phase, primarily responsible for the translucency of the material. Polycrystalline ceramics are basically characterized by having a larger amount of crystalline phase as alumina, leucite, lithium disilicate and zirconia ${ }^{3}$.

Improvements in the clinical performance of ceramic restorations are closely related with the use of adhesive resin materials for luting procedures, including a photoactivation method and efficiency of the adhesive system ${ }^{4}$. The use of resin cements is preferred because they offer many advantages, as the ability to micromechanical bond to tooth structure, low solubility and greater strength to wear compared to conventional dental cements ${ }^{5}$. The resin cements are divided into conventional and self-adhesive materials and involve two bonding interfaces: tooth structure/resin cement and resin cement/restorative material ${ }^{6}$. The conventional resin cements require prior treatments of the tooth surface. The bonding of these materials to the tooth is dependent of an adhesive system, following the same principles of direct composite resin restorations. The bonding of the resin cement to the restoration depends on the type of ceramic material and the treatments performed on the internal surface which include roughness with a diamond bur, sandblasting with aluminum oxide, etching with hydrofluoric acid, ammonia bifluoride or acidulated phosphate fluoride, silanization and ultrasonic cleaning ${ }^{7}$. This total-etch adhesive protocol of conventional resin cements is complex, require multiple steps and may make the effectiveness of bonding vulnerable 8 .

The self-adhesive resin cements were developed to simply the bonding procedures, once do not require any pre-treatment of dental substrate, reduces clinical steps and the "window of contamination ${ }^{\prime \prime, 9}$. The cementation can be accomplished in a single step ${ }^{8}$. The organic matrix of these materials consists of a multifunctional monomer (generally a phosphoric acid ester methacrylate). This can react with the filler of the resin cement as well as hydroxyapatite of dentin and ename ${ }^{10}$. Following the trend to simplify the technique of luting, many manufacturers launched their versions for the self-adhesive cements.

Usually most of scientific papers that involve the study of indirect restorative materials such as dental ceramics and their adhesive procedure focus in the investigation of the effectiveness of these adhesive materials to bond in tooth substrates, with few studies focusing the other bonding interface between resin materials and dental ceramics. Thus, the present study aimed to evaluate the microshear bonding strength between conventional and self-adhesive resin cements to dental ceramics, 24 hours and 6 months after the bonding procedure. Two null hypotheses were tested: (1) different resin cements did not have difference in the bonding strength to dental ceramics and (2) the bonding strength of resin cements to dental ceramics measured after 6 month of bonding procedure did not have difference for the measurements done after 24 hours. 


\section{Materials and Methods}

Twenty discs of Starlight feldspathic ceramic (DeguDent Gmbh, Hanau-Wolfgang, Germany) were made using a silicone matrix, measuring $15 \mathrm{~mm}$ in diameter and $2 \mathrm{~mm}$ in thickness. A Centurion furnace (Degussa-Ney Dental, Yucaipa, CA, USA) was used for the ceramics sintering, following the time-temperature scale recommended by the manufacturer. After sintering, the samples were finished and polished with Ninja points (Talladium do Brasil, Curitiba, PR, Brazil), \#104023 diamond points flame-shaped and \#35 inverted cone-shape. The measurements of the samples were checked with a digital caliper (Mitutoyo, Suzano, SP, Brazil).

One of the ceramic surfaces was etched with 10\% hydrofluoric acid (Porcelain conditioner, Angelus, Londrina, PR, Brazil) during 2 minutes. After the acid etching, the specimens were washed with air-water spray during 60 seconds and dried with compressed air for 30 seconds, and then submitted to the silanizing agent application (Ceramic Primer, 3M Espe, St. Paul, MN, USA) during 1 minute.

The discs were divided into four groups, according to the resin cement tested $(n=5)$. The bonding process of resin cements to ceramic was performed by the insertion of resin cements in Tygon®-Microbore (TGY-030, Small Parts Inc., Miami Lakes, FL, USA) tubes with $0.7 \mathrm{~mm}$ of internal diameter, $2.1 \mathrm{~mm}$ of external diameter and $1.0 \mathrm{~mm}$ of height, located on the prepared surface of the ceramic. Four resin cements were used: RelyX ARC (3M Espe) and Panavia F (Kuraray, Kurashiki, Japan) conventional resin cements, RelyX Unicem (3M Espe) and seT (SDI, Bayswater Victoria, Australia) self-adhesives resin cements. Six tubes of each cement were placed in each ceramic specimen.

The photoactivation of cement was performed using Ultraled II light-curing unit (Dabi Atlante, Ribeirão Preto, SP, Brazil), with $700 \mathrm{~mW} / \mathrm{cm}^{2}$ light output intensity for 40 seconds, through the ceramic surface. The photoactivation process was conducted in a darkened environment, so the external ambient light has not influenced the degree of conversion of the resin cement. The specimens were stored in deionized water at $37^{\circ} \mathrm{C}$ for 24 hours.

The specimens were submitted to microshear bonding test in a universal testing machine (DL 3000, Emic, São José dos Pinhais, SP, Brazil) at a crosshead speed of $1.0 \mathrm{~mm} / \mathrm{min}$. For the test, each specimen was horizontally positioned in a metal glove and an orthodontic wire with $0.3 \mathrm{~mm}$ forming a loop surrounding the cylinder of resin cement composite has performed the tensile stress resulting in a microshear bonding test. The microshear bonding strength ( $\mathrm{MPa}$ ) was calculated by: F/A where: $F$ was the force applied $(\mathrm{N})$; and $\mathrm{A}$, the area of bonding $\left(\mathrm{mm}^{2}\right)$.

To perform the microshear bonding strength after 6 months, the same ceramic discs were worn with no. 80-, 320- and 600-grit silicon carbide paper (Extec Corp, Enfield, CT, USA) and then submitted to same surface treatment with hydrofluoric acid and silane application. Resin cements were positioned in Tygon®-Microbore tubes as the same manner as above described. The specimens were stored in deionized water at $37^{\circ} \mathrm{C}$ for 6 months, changed weekly, until the microshear bonding test.

Data were submitted to the Kolmorov-Smirnov normality test and the averages compared by ANOVA and Fisher's PLSD test $(p=0.05)$. Two factors were studied: material (RelyX ARC, Panavia F, RelyX Unicem and seT) and time (24 hours and 6 months). 


\section{Results}

ANOVA (Table 1) showed that there was a statistically significant difference between the cements studied $(p<0.001)$ as well as between the evaluated times $(p<0.001)$. The interaction between the groups was not statistically significant $(p=0.94)$.

Table 1. ANOVA results for microshear bonding strength (MPa).

\begin{tabular}{lccccccc}
\hline & DF & Sum of squares & Mean Square & F-Value & P-Value & Lambda & Power \\
\hline Groups & 3 & 1191.924 & 397.308 & 34.491 & $<.0001$ & 103.472 & 1.000 \\
\hline Time & 1 & 318.310 & 318.310 & 27.633 & $<.0001$ & 27.633 & 1.000 \\
\hline Groups * time & 3 & 4.499 & 1.500 & .130 & .9414 & .391 & .071 \\
\hline Residual & 31 & 357.099 & & & & & \\
\hline
\end{tabular}

At 24 hours, RelyX ARC showed the highest microshear bonding strength without statistical difference to RelyX Unicem and $\mathrm{seT}(\mathrm{p}>0.05)$. The lowest values were obtained for Panavia $F$ with significant difference for the other cements $(p<0.001)$. These data are shown in Table 2. The same phenomenon occurred to the specimens analyzed 6 months after the bonding procedure, in which Panavia F showed the lowest value of microshear bonding strength, compared to the other cements studied.

Table 2. Means of microshear bonding strength (MPa) between the resin cements and feldspathic ceramic, 24 hours and 6 months after the bonding procedure.

\begin{tabular}{lcccc}
\hline \multicolumn{4}{c}{ Microshear bonding strength (MPa) } \\
\hline RelyX ARC & Panavia & RelyX Unicem & Set \\
\hline 24 hours & $21.13 \pm 4.77 \mathrm{~A} \mathrm{a}$ & $8.40 \pm 3.75 \mathrm{~A} \mathrm{~b}$ & $20.86 \pm 0.43 \mathrm{~A} \mathrm{a}$ & $19.12 \pm 1.98 \mathrm{~A} \mathrm{a}$ \\
\hline 6 months & $26.54 \pm 5.45 \mathrm{~A} \mathrm{a}$ & $13.18 \pm 2.92 \mathrm{~B} \mathrm{~b}$ & $27.11 \pm 2.81 \mathrm{~B} \mathrm{a}$ & $25.60 \pm 2.06 \mathrm{~B}$ a \\
\hline
\end{tabular}

Distinct letters, uppercase in the column and lowercase letter in the line, show a statistically significant difference between them $(p<0.05)$.

A significant increase in the values of microshear bonding strength was found 6 months after the bonding procedure compared to the 24 hour for all resin cements studied (Table 2). Just for RelyX ARC, this increase was not statistically significant ( $p=0.134)$.

\section{Discussion}

The success of the luting procedure is dependent of a strong and durable bonding strength in both interfaces between resin cement to ceramics and between resin cement to tooth ${ }^{11}$. In this study, the focus was to evaluate the bonding strength in the resin cement-ceramic interface. Currently, the bonding process between feldspathic ceramic to resin cements is provided by etching the ceramic surface with hydrofluoric acid, followed by silane agent application. Both have the property to increase the wetting of the cement on the surface, facilitating the contact between the materials. Fur- 
thermore, the silane performs a chemical bonding between the silica contained in ceramic and the organic matrix of resin cements, through siloxane bonding ${ }^{12,13}$. For this reason, all ceramic specimens received the same surface treatment, based on application of hydrofluoric acid followed by silanization.

Based on the results of this study, it was found that Panavia F showed the lowest value of microshear bond strength in both periods studied (Table 2). In the other hand, RelyX ARC showed the highest value of microshear bond strength after 24 hours and 6 months, without significant difference to RelyX Unicem and seT cements. The difference in the behavior of conventional cements could be related to the formulation and their viscosity. The difference in composition is inherent to the materials and could not be compensated by the polymerization, although an increased viscosity of the material could restrict the photoactivation process ${ }^{14}$. The conventional cements contain in their basic composition, resin monomers as BIS-GMA (bisphenol A glycidyl methacrylate) or UDMA (urethane dimethacrylate). Self-adhesive resin cements have in their composition phosphoric acid monomers as MDP or META ${ }^{15}$. Kern and Thompson $^{16}$ (1995) related in their studies that MDP-modified resin cements, as Panavia $F$, has better adhesion to aluminized surfaces than conventional resin cements based on BIS-GMA (as RelyX ARC), because of the existence of chemical bonding between MDP and alumina. Furthermore, phosphate ester groups of MPD-modified resin cement have been described in the literature as able to bond directly to metal oxides ${ }^{17}$. However, this benefit may be limited when it bonds to feldspathic ceramics, as used in this study. The high viscosity shown by Panavia F could not allow sufficient flow, negatively affecting the bonding to ceramics, as shown also in dentin interface ${ }^{18}$. This could probably explain the lower values found for Panavia F in this study.

Regarding the self-adhesive resin cements, RelyX Unicem and seT did not show significant differences between them, but showed higher values of bonding strength than Panavia F in both analyzed periods (Table 2). One of the factors that could explain the performance of the self-adhesive is the way of commercial presentation. They are available in the market in capsules, which require pre-activation, and need a mechanical device for mixing procedure, with no manual mixing. The mechanical mixing enables greater effectiveness for the cement, once the manual manipulation could lead the formation and entrapment of air bubbles creating voids in the adhesive interface and therefore, interference in the performance of the material ${ }^{19}$.

The analysis of the microshear bonding strength after 6 months showed a statistically significant increase in the values compared to the values after 24 hours for all cements studied, except for RelyX ARC (Table 2). The late polymerization may be the main responsible for the increase of the bonding strength over time, once the photoactivation provides the generation of free radicals responsible for the induction of chemical polymerization ${ }^{20}$. According to Arrais et al. ${ }^{21}$ (2009), the initial exposure to light may cause a change in the viscosity of dual-cured materials, making difficult the migration of active radicals, which could lead to a delay in the process of polymerization.

This study has showed some limitations as the difficulty of load standardization applied during the resin cement insertion inside the microtubes representing the pressure during the luting procedure, the fixation of the tube so the cement could 
be adequately inserted and the removal of the Tygon®-Microbore tubes before the microshear bonding test. Other aging process as thermal and mechanical cycling, are also necessary to guarantee the longevity of the bonded interface studied ${ }^{22}$, since in the present study, only the storage in water was realized. However, it could be seen that the inherent properties of the resin cement affects the final performance of the bonding interface.

\section{Conclusion}

Based on the results, it can be observed that time had a directly influence in the bonding strength between resin cement and feldsphatic ceramic. For all resin cements, except for RelyX ARC, there was an increase of microshear bonding strength over time. Panavia F resin cement showed the lowest value of microshear bonding strength compared to the other resin cements in both times analyzed. The characteristics of these materials have influenced its short and long-term performance.

\section{Acknowledgements}

This study was supported by FAPESP (2009/17826-3).

\section{Conflicts of interest}

The authors declare no conflict of interest.

\section{References}

1. Seemann R, Flury S, Pfefferkornb F, Lussia A, Noackc MJ. Restorative dentistry and restorative materials over the next 20 years: A Delphi survey. Dent Mater. 2014 Apr; 30(4):442-8. doi: 10.1016/j.dental.2014.01.013.

2. Nayar S, Aruna U, Bhat WM. Enhanced aesthetics with all ceramics restoration. J Pharm Bioallied Sci. 2015 Apr;7(Suppl 1):S282-4. doi: 10.4103/0975-7406.155957.

3. Kim RJ, Woo JS, Lee IB, Yi YA, Hwang JY, Seo DG. Performance of universal adhesives on bonding to leucite-reinforced ceramic. Biomater Res. 2015 May;19:11. doi: 10.1186/s40824-015-0035-1.

4. Kuguimiya RN, Rode KM, Carneiro PM, Aranha AC, Turbino ML. Influence of Curing Units and Indirect Restorative Materials on the Hardness of Two Dual-curing Resin Cements Evaluated by the Nanoindentation Test. J Adhes Dent. 2015 Jun;17(3):243-8. doi: 10.3290/j.jad.a34399.

5. Tian T, Tsoi JK, Matinlinna JP, Burrow MF. Aspects of bonding between resin luting cements and glass ceramic materials. Dent Mater. 2014 Jul;30(7):e147-62. doi: 10.1016/j.dental.2014.01.017.

6. Pavan S, dos Santos PH, Berger S, Bedran-Russo AK. The effect of dentin pretreatment on the microtensile bond strength of self-adhesive resin cements. J Prosthet Dent. 2010 Oct;104(4):258-64. doi: 10.1016/S0022-3913(10)60134-5.

7. Leite FP, Özcan M, Valandro LF, Moreira CH, Amaral R, Bottino MA et al. Effect of the etching duration and ultrasonic cleaning on microtensile bond strength between feldspathic ceramic and resin cement. J Adhes. 2013:89(3):159-73

8. Bellan MC, Cunha PFJSD, Tavares JG, Spohr AM, Mota EG. Microtensile bond strength of CAD/ CAM materials to dentin under different adhesive strategies. Braz Oral Res. 2017 Dec 18;31:e109. doi: 10.1590/1807-3107BOR-2017. 
9. Reich SM, Wichmann M, Frankenberger R, Zajc D. Effect of surface treatment on the shear bond strength of three resin cements to a machinable feldspatic ceramic. J Biomed Mater Res B Appl Biomater. 2005 Aug;74(2):740-6.

10. Hikita K, Van Meerbeek B, De Munck J, Ikeda T, Van Landuyt K, Maida T, et al. Bonding effectiveness of adhesive luting agents to enamel and dentin. Dent Mater. 2007 Jan;23(1):71-80.

11. Gilbert S, Keul C, Roos M, Edelhoff D, Stawarczyk B. Bonding between CAD/CAM resin and resin composite cements dependent on bonding agents: three different in vitro test methods. Clin Oral Invest. 2015 Mar;20(2):227-36. doi: 10.1007/s00784-015-1494-4.

12. Della Bona A, Anusavice KJ, Shen C. Microtensile strength of composite bonded to Hot-pressed Ceramics. J Adhes Dent. 2000 Winter;2(4):305-13.

13. Lu R, Harcourt JK, Tyas MJ, Alexander B. An investigation of composite resin/porcelain interface. Aust Dent J. 1992 Feb; 37(1):12-9.

14. Fonseca AS, Mizrahi J, Menezes LR, Valente LL, de Moraes RR, Schneider LF. The effect of time between handling and photoactivation on self-adhesive resin cement properties. J Prosthodont. 2014 Jun;23(4):302-7. doi: 10.1111/jopr.12108.

15. Jardel V, Degrange M, Picard B, Derrien G. Surface energy of etched ceramic. Int J Prosthodont. 1999 Sep-Oct;12(5):415-8.

16. Kern M, Thompson VP. Bonding to glass infiltrated alumina ceramic: adhesive methods and their durability. J Prosthet Dent. 1995 Mar;73(3):240-9.

17. Fabiao MM, Stape TH, Yanikian CR, de Lima AF, Pizi EC, Baron GM, et al. Influence of different adhesive protocols on ceramic bond strength and degree of conversion of resin cements. Int J Adhes Adhesives. 2015 Oct;62:7-13.

18. Suzuki TY, Godas AL, Guedes AP, Catelan A, Pavan S, Briso AL, et al. Microtensile bond strength of resin cements to caries-affected dentin. J Prosthet Dent. 2013 Jul; 110(1):47-55. doi: 10.1016/S0022-3913(13)60339-X.

19. Aksornmuang J, Nakajima M, Foxton R, Tagami J. Mechanical properties and bond strength of dualcure resin composites to root canal dentin. Dent Mater. 2007 Feb;23(2):226-34.

20. Moraes RR, Brandt WC, Naves LZ, Sobrinho LC, Piva E. Light-and time-dependent polymerization of dual-cured resin luting agent beneath ceramic. Acta Odontol Scand. 2008 Oct;66(5):257-61. doi: $10.1080 / 00016350802241563$.

21. Arrais CAG, Giannini M, Rueggeberg FA. Kinetic analysis of monomer conversion in auto- and dualpolymerizing modes of commercial resin luting cements. J Prosthet Dent. 2009 Feb;101(2):128-36. doi: 10.1016/S0022-3913(09)60008-1.

22. Blumer L, Schmidli F, Weiger R, Fischer J. A systematic approach to standardize artificialaging of resin composite cements. Dent Mater. 2015 Jul;31(7):855-63. doi: 10.1016/j.dental.2015.04.015. 